\title{
Sustaining Agile Beyond Adoption
}

\author{
Leonor Barroca \\ The Open University \\ Milton Keynes, MK7 6AA \\ UK \\ leonor.barroca@open.ac.uk
}

\author{
Peggy Gregory \\ University of Central Lancashire \\ Preston PR1 2HE \\ UK \\ ajgregory@uclan.ac.uk
}

\author{
Kati Kuusinen \\ University of Southern Denmark \\ Odense \\ Denmark \\ kaku@mmmi.sdu.dk
}

\author{
Helen Sharp \\ The Open University \\ Milton Keynes, MK7 6AA \\ UK \\ helen.sharp@open.ac.uk
}

\author{
Raid AlQaisi \\ University of Central Lancashire \\ Preston PR1 2HE \\ UK \\ ralqaisi@uclan.ac.uk
}

\begin{abstract}
Agile approaches are adopted in industry to improve outcomes from software development, and are increasingly the subject of research studies. However, adoption is not the end of the story. Agile requires on-going change and commitment in order to become sustainable and embedded within teams and organisations. This study explores current perceptions of post-adoptive agility. We asked 50 practitioners 'what does agile sustainability mean to you?'. Analysis of practitioner comments identified four themes: being completely agile, independent, focused on business value and need, and consistent across time. Post-adoptive agile is an under-researched area, there is inconsistent use of terminology, and there is a gap between practitioners' and researchers' perceptions about what is important for sustaining agile.
\end{abstract}

Keywords-agile sustainability; post-adoptive agile use; practitioner study; agile methods

\section{INTRODUCTION}

Agile working faces many challenges in practice. As observed by Gregory et al. [1], the landscape of such challenges is complex and intertwining, with some challenges persisting even when research has been carried out in the area, while others change focus over time. One area that has changed focus is the need to 'sustain' agility. In the early years of agile software development, it was often lamented by practitioners that a successful agile team could easily lose agility once the project had ended and team members returned to their usual working environment. However, the focus of research at that time was on understanding agile adoption and agile practices. Now, the challenges surrounding the practices of agile teams and projects are better understood, and extensive support exists to aid the adoption of agile from both research and practice sources.

Recently, agile has spread more widely to larger projects and indeed has extended across the organisation into non-IT areas. The need to maintain agility remains, but the effort has changed focus from adoption to its continuous improvement [2]. Organisations are realising that the benefits that agile brings to software teams, mostly in ways people work and the culture they develop, can also benefit areas of the organisation beyond IT. In particular, the concept of agility is seen as a solution to address problems faced by an uncertain, quickly changing competitive environment [3]. It is therefore important for organisations to understand how agile can be sustained and improved before they take decisions on extending it to the whole enterprise.

In this paper 'sustaining agile' is about supporting and evolving agile once adopted and encouraging its principles to be kept alive, sometimes in a non-agile environment, without it succumbing to bureaucratic fossilisation. Agile requires ongoing change, improvement and commitment in order to become sustainable and embedded within teams and organisations. The goal of this paper is to explore the concept of 'sustaining agile' via a poll of practitioners' views. The main contribution of this paper is twofold: for researchers, it highlights areas that are of concern to practitioners but not the focus of research; for practitioners, it highlights success factors reported to support agile sustainability. Another contribution is that practitioners' views of sustaining agile focus on principles, mind-set and being agile across the whole organisation rather than exclusively on success factors. The paper sets the scene for further research needed to bridge the well-recognised gap between research and practice, here in sustaining agile beyond adoption.

The rest of the paper is structured as follows. Section II defines sustainability as discussed in this paper. Section III discusses the motivation for the paper, and Section IV describes the practitioners' poll we conducted. We discuss the results in section $\mathrm{V}$ in the context of a systematic literature review on sustainability, and section VI presents conclusions.

\section{What IS SUSTAINABILITY?}

Sustainability has been investigated from various angles in software engineering and the term 'sustainability' is used with different meanings. The United Nations definition of sustainable development [4] has been applied to software [5] and to software development [6] to include societal, economic, environmental and human dimensions with regards to the production and usage of software systems including development and maintenance processes [7].

The perspective we take on sustaining agile differs from those above and is inline with that of Senapathi and Srinivasan [8]. They view sustaining agile with regards to the six stages presented in the diffusion of innovation theory by Rogers [9]. 
Thus, they see sustainability from a change management point of view and consider it as the post-adoptive stages where a change is accepted, routinised and infused into an organisation. These are the three final phases of Rogers' diffusion of innovation theory where, in this case, agile is being used, it becomes normalised, and it penetrates deeply and widely in the organisation. Sedano et al. [10] investigate sustainability on development team level. They define sustainable software development as the "ability and propensity of a software development team to mitigate the negative effects of major disruptions, especially team churn, on its productivity and effectiveness".

We adopt Rogers' view and use the term 'sustainability' as in Senapathi and Srinivasan [11], where the main concern is about what happens with agile once it has been adopted and accepted and becomes continuously and extensively used. However, our view on sustainability goes beyond Rogers' because of the need for continuous improvement once agile is adopted. Thus, sustainability in the context of agile refers to the ability to support and evolve agile once accepted, encouraging its continuous improvement.

\section{Motivation}

\section{A. Challenge Wall}

We developed a Challenge Wall to collect agile challenges from a diverse range of practitioners. We took this Challenge Wall to five agile conferences and events between October 2013 and October 2014 and carried out a thematic analysis of the data collected.

As a result of this thematic analysis we identified seven themes and 27 subthemes from a set of 190 challenges. The seven themes were: Claims and Limitations, Organisation, Culture, Teams, Sustainability, Scaling, and Value. Within these, two were particularly distinct with a high number of challenges. Claims and Limitations had 46 challenges, mostly about misconceptions, shortcomings, and hype. Organisation had 44 challenges, mostly about business concerns; of these 44 challenges only four were about adoption.

At the time we carried out this analysis, we identified a Sustainability theme with, among others, specific process improvement challenges related to the need for continuous change and commitment to make agile sustainable and embedded within teams and organisations. However, looking at the 190 challenges through a sustainability lens, it became apparent that many of the challenges we collected related to the post-adoption and continuous improvement of agile and that sustainability, as defined above, runs through many of them, mainly those under the themes of Organisation, Culture, Teams, Scaling and Value. Most of the subthemes under Organisation are about how to keep agile going, be it addressing the conflict between business and IT transformation, or obtaining management buy-in and understanding, or making agile work within a non-agile environment, or obtaining commitment and engagement. Under Culture, subthemes such as organisational culture and changing mindsets are also relevant to keeping agile and improving it. Teams, Scaling and Value (business value and measurement) are also under the umbrella of sustaining agile, across multiple teams, in large projects and within the organisation.

\section{B. The search/need for sustainability is pervasive}

The authors are part of the Agile Research Network (ARN) which is a collaboration between researchers at two UK universities investigating Agile as it matures and becomes mainstream. The ARN has conducted a series of case studies (www.agileresearchnetwork.org) working with practitioners, bridging the gap between research and practice. Although it was not explicit from the outset, most of the case studies we have carried out have an underlying challenge related to agile sustainability. In fact, in our experience, practitioners are nowadays less concerned about adopting agile and more concerned with sustaining agile [1]. Furthermore, we claim that sustaining agile is still an understudied topic, as it was a decade ago.

Senapathi and Srinivasan [11] carried out a systematic literature review of sustained agile usage, with the objective of understanding factors that affect sustained usage of agile methods. They identify 14 factors: compatibility, relative advantage, management support, methodology champion, attitude, motivation, team composition, team empowerment, training, agile mindset, technical competence, agile engineering practices, documentation and tool support. They also find that multiple success factors in the right balance and combination are needed for sustained agile usage. They also presented a structural equation modelling study of factors that affect agile usage [12]. The strongest predictors for continued agile usage after the adoption phase are relative advantage of agile and having agile coaches. They also found that the intensity and extent of the use of agile have an impact on effectiveness of agile measured by predictability, productivity, quality and customer satisfaction.

\section{PRACTITIONERS' Views}

We asked practitioners about their understanding of 'agile sustainability' so we could explore different interpretations of the concept. As sustaining agile is ultimately of practical importance, it was essential that we understood the views of practitioners.

\section{A. Method}

To understand practitioners' views we collected sticky note comments on a whiteboard at two conference venues asking the question 'What does agile sustainability mean to you?'. Thirty-five sticky notes were collected at XP2016, the International Conference on Agile Software Development, which had 380 attendees of which $70 \%$ were practitioners and $25 \%$ were academics ( $5 \%$ other), and 15 were collected at ABC2016, the UK Agile Business Conference, which had 294 attendees of which $93 \%$ were practitioners and $7 \%$ were academics, totalling 50 responses.

Two researchers independently analysed the data using thematic analysis to explore participants' experience, perceptions, and views. The analysis started by reading and 
coding each individual sticky note, then grouping the codes into sub-themes, and finally grouping the sub-themes into themes [13]. These were reviewed and a final analysis agreed upon.

\section{B. Findings}

The themes and sub-themes are shown in Table 1 below.

TABLE I: THEMATIC ANALYSIS OF PRACTITIONERS' COMMENTS

\begin{tabular}{ll}
\hline Themes & Sub-themes \\
\hline Completely agile & Whole organisation \\
& Mind-set \\
& Principles \\
Independent & Continuous learning \\
& Self-sufficient \\
Focused on business value and need & User/customer \\
& Value \\
& Business \\
& Appropriate use \\
Consistent across time & Energy, pace, success \\
& Technical \\
\hline
\end{tabular}

\section{1) Completely agile}

This consisted of 15 sticky note comments, with three subthemes: whole organisation, mind-set, and principles. The first was about rolling agile out across the wider organisation or scaling it up, for example: "Continuing the ideas throughout the company and between developing/expanding teams with variable levels of experience" and "Roll out to the whole organisation". The second was about taking on an agile mindset, for example "Staying truly agile, not falling back on old habits/traditional development. Getting to agile mind-set". The third was about emphasizing agile principles, for example "Focus on principles and people, not methods or tools".

\section{2) Independent}

This theme contained 14 sticky notes. The two sub-themes were learning and self-sufficiency, with the link between the two being that continuous learning results in adaptation to change, and this in turn results in self-sufficiency. Learning was mentioned in nine sticky notes, for example "Keep learning and making changes to become better adapted to an always changing environment" Self-sufficiency was exemplified by the comment "If you walk away, it keeps going".

\section{3) Focused on business value and need}

This theme included 13 sticky notes and four sub-themes: user/customer need, value, business need, and appropriate use. These all relate to the importance of prioritising activities that align most closely with the businesses core goals and user needs. Examples include: "Working on user stories/requirements instead of focusing on technologies", "Predictably delivering value and delight", and "Sustain[ing] agile means predictable business on and on". Finally, the fourth theme

4) Consistent across time
This contained seven sticky notes and two sub-themes. The energy/pace/success sub-theme contained comments about sustainable pace, and leadership for example "Sustainable pace: no hero programming, work/life balance, celebrate success" and "Active leadership and trust". The technical subtheme suggested technical capabilities for example "Attention to technical debt".

The four themes identified from this exercise read like a high-level description of what is important about the agile approach. This is unsurprising because we asked a general question rather than a context-specific one. They provide a contrast with many papers in Senapathi and Srinnivasan's literature review [11] which describe success factors in postadoptive agile. However, these sticky note themes resonate with work [14] on how agile can be more widely adopted outside the IT area and how its principles can be applied in many different contexts.

\section{DISCUSSION}

In this paper we explore agile sustainability using a practitioner poll. The key themes from the poll are that sustainability in agile is about being completely agile, independent, focused on business need and value, and consistent over time. Practitioners emphasise agile concepts and principles in the round while existing research focuses instead on success factors [11]. This is an important finding that suggests researchers are not focussing on issues that are important to practitioners. Although success is implicit in the practitioners' viewpoint through the focused on business value and need, it is not directly mentioned.

Culture change is acknowledged in the practitioners' poll, through the completely agile theme and resonates with some of the success factors in [11] such as attitude and agile mindset.

Whole organisation is a sub-theme that includes comments about rolling out agile beyond IT in organisations. This is an area with increasing research interest $[14,16]$ that did not appear in the Senapathi and Srinivasan literature review. Fitzgerald and Stol [14] highlight the need to establish a link between business strategy and software development, and coin it as BizDev. They argue that continuity is needed between discrete phases of software development, from planning through to use, underpinned by continuous trust, monitoring, improvement and innovation. This suggests a more holistic approach beyond software, extending agile to the wider organisation.

Our findings suggest that research needs to go much wider in investigating how agile may be sustained. Without some commitment from the whole organisation, without understanding what business value means at all levels and without developing people, culture and mind-sets the benefits of agile cannot be continuously sustained and improved. Sustaining agile has started to gather interest within the research community [8], [16]. The views from practitioners suggest that the context where agile is supported and sustained and the overall culture are crucial. There is a need for research to take a broader view to understanding how the challenges 
encountered in sustaining agile can be addressed in the wider context of organisations.

The limitations of this study relate to the practitioners' poll and the need to look more systematically at the more recent literature. The practitioner poll was carried out in an international conference, XP 2016, and a UK based one, ABC 2016. While $\mathrm{ABC}$ has a very high percentage of practitioners $(93 \%), 25 \%$ of XP attendees are academics. We cannot therefore guarantee that all answers are from practitioners. The poll is a snapshot and cannot be considered widely representative due to the small number (50) of sticky notes collected. However, these findings are indicative of the importance of this area of research and the need for a much wider investigation. The systematic literature review that we used was published in 2013 and although we looked at more recent literature we need to complement this study with a systematic mapping of more recent research.

\section{CONCLUSIONS}

In this paper we have explored how agile approaches for software development are sustained beyond their adoption. We investigated the topic by talking to practitioners about what agile sustainability means to them and scanned existing literature. In summary we found the following:

- there is little research literature exploring the phenomenon.

- $\quad$ practitioners viewed sustainability as being completely agile, independent, focused on business value, and consistent over time rather than being exclusively about success factors.

- the term 'sustainability' is not widely used in the literature, other terms used include post-adoption, routinisation, and maturity.

We conclude that there is a gap between the way practitioners view agile sustainability and the way it is being investigated by researchers. We suggest that there is a need for this research topic to be further developed and for research studies to investigate agile sustainability through the lens of being completely agile, independent, focusing on business value and users, and consistency across time.

\section{REFERENCES}

[1] P. Gregory, L. Barroca, H. Sharp, A. Deshpande, and K. Taylor, 'The challenges that challenge: Engaging with agile practitioners' concerns.', Inf. Softw. Technol., vol. 77, pp. 92-104, 2016.

[2] T. Dingsøyr and C. Lassenius, 'Emerging themes in agile software development: Introduction to the special section on continuous value delivery', Inf. Softw. Technol., vol. 77, pp. 56-60, 2016.

[3] T. Karvonen, H. Sharp, and L. Barroca, 'Enterprise agility: Why is transformation so hard?', in XP 2018 (to appear), 2018.
[4] UN, 'Report of the World Commission on Environment and Development: Our Common Future', in United Nations Conference on Environment and Development, 1987.

[5] M. Dick, S. Naumann, and N. Kuhn, 'A model and selected instances of green and sustainable software', IFIP Adv. Inf. Commun. Technol., vol. 328, pp. 248259, 2010.

[6] M. Dick and S. Naumann, 'Enhancing software engineering processes towards sustainable software product design', Integr. Environ. Inf. Eur., vol. 2010, pp. 706-715, 2010.

[7] B. Penzenstadler, V. Bauer, and C. Calero, 'Sustainability in software engineering: A systematic literature review', in 16th International Conference on Evaluation \& Assessment in Software Engineering (EASE 2012), 2012, no. January 2012.

[8] M. Senapathi and A. Srinivasan, 'Understanding postadoptive agile usage: An exploratory cross-case analysis', J. Syst. Softw., vol. 85, no. 6, pp. 12551268, 2012.

[9] E. Rogers, Diffusion of Innovations, 5th Edition. Simon and Schuster, 2010.

[10] T. Sedano, P. Ralph, and C. Péraire, 'Sustainable Software Development Through Overlapping Pair Rotation', in Proceedings of the 10th ACM/IEEE International Symposium on Empirical Software Engineering and Measurement, 2016, p. 19:1-19:10.

[11] M. Senapathi and A. Srinivasan, 'Sustained agile usage: A Systematic Literature review', in Proceedings of the 17th International Conference on Evaluation and Assessment in Software Engineering EASE '13, 2013, p. 119.

[12] M. Senapathi and A. Srinivasan, 'An Empirical Investigation of the Factors Affecting Agile Usage', in Proceedings of the 18th International Conference on Evaluation and Assessment in Software Engineering, 2014, pp. 1-10.

[13] J. Ritchie, J. Lewis, C. M. Nicholls, and R. Ormston, Qualitative research practice: A guide for social science students and researchers. Sage, 2013.

[14] B. Fitzgerald and K.-J. Stol, 'Continuous software engineering and beyond: trends and challenges', in Proceedings of the 1st International Workshop on Rapid Continuous Software Engineering - RCoSE 2014, 2014, pp. 1-9.

[15] B. Fitzgerald and K. J. Stol, 'Continuous software engineering: A roadmap and agenda', J. Syst. Softw., vol. 123, pp. 176-189, 2017.

[16] M. Senapathi and M. L. Drury-Grogan, 'Refining a model for sustained usage of agile methodologies', $J$. Syst. Softw., vol. 132, pp. 298-316, 2017. 